\title{
Low-energy properties of anisotropic two-dimensional spin-1/2 Heisenberg models in staggered magnetic fields
}

\author{
Bin Xi, ${ }^{1,2,3}$ Shijie $\mathrm{Hu},{ }^{4,1}$ Jize Zhao, ${ }^{5,6}$ Gang $\mathrm{Su}^{3}{ }^{3}$ B. Normand, ${ }^{1}$ and Xiaoqun Wang ${ }^{1}$ \\ ${ }^{1}$ Department of Physics, Renmin University of China, Beijing 100872, China \\ ${ }^{2}$ Institute of Theoretical Physics, Chinese Academy of Sciences, Beijing 100190, China \\ ${ }^{3}$ College of Physical Sciences, Graduate University of the Chinese Academy of Sciences, Beijing 100049, China \\ ${ }^{4}$ Institut für Theoretische Physik, Georg-August-Universität Goettingen, 37077 Göttingen, Germany \\ ${ }^{5}$ Institute for Solid State Physics, University of Tokyo, Kashiwa, Chiba 277-8581, Japan \\ ${ }^{6}$ Department of Physics and Astronomy, California State University, Northridge, CA 91330, USA
}

(Dated: October 11, 2018)

\begin{abstract}
We present a systematic study of the anisotropic spin-1/2 Heisenberg model in staggered magnetic fields in two dimensions (2D). To mimic real materials, we consider a system of coupled, antiferromagnetic chains, whose interchain interaction can be either ferro- or antiferromagnetic. When the staggered field is commensurate with the magnetic interactions, an energy gap opens immediately and follows a power law as a function of the applied field, similar to the situation in 1D. When the field competes with the interactions, a quantum phase transition (QPT) occurs from a gapless, magnetically ordered phase at low fields to a gapped, disordered regime. We use a continuous-time Monte Carlo method to compute the staggered moment of the ordered phases and the spin gap of the disordered phases. We deduce the phase diagrams as functions of the anisotropy ratio and the applied field, and calculate the scaling behavior of the models in both quantities. We show that in the competitive case, the staggered field acts to maintain a regime of quasi-1D behavior around the $\mathrm{QPT}$, and we discuss as a consequence the nature of the crossover from $1 \mathrm{D}$ to $2 \mathrm{D}$ physics.

PACS numbers: 75.10.Jm, 75.30.Kz, 75.40.Cx, 75.40.Mg
\end{abstract}

\section{INTRODUCTION}

The spin- $1 / 2$ antiferromagnetic Heisenberg model in a staggered magnetic field has attracted increasing interest as the number of real materials it describes continues to rise. While the model in zero field is gapless in all dimensions, the staggered field lowers symmetries, changes universality classes, and induces gapped states and unconventional, gapped elementary excitations. Intensive investigations into staggered-field effects in quantum magnets were motivated originally by inelastic neutron scattering experiments on the quasi-1D spin chain copper benzoate $\underline{\underline{1}}$ Application of a uniform magnetic field to this material caused an unexpected gap to open in the excitation spectrum. This energy gap follows a power law as a function of the applied magnetic field, but its magnitude depends strongly on the direction of the applied magnetic field.

This unusual experimental finding was soon explained $2, \underline{3}$ by the fact that copper benzoate is not a perfect chain, but has an alternating crystal structure giving rise to a staggered gyromagnetic tensor, possibly accompanied by a Dzyaloshiskii-Moriya (DM) interaction. The full Hamiltonian in a uniform external magnetic field is

$$
\begin{array}{r}
\hat{H}=\sum_{i}\left[J \hat{\mathbf{S}}_{i} \cdot \hat{\mathbf{S}}_{i+1}-(-1)^{i} \mathbf{D} \cdot \hat{\mathbf{S}}_{i} \times \hat{\mathbf{S}}_{i+1}\right. \\
\left.-\mu_{B} \mathbf{H} \cdot\left(\mathbf{g}^{u}+(-1)^{i} \mathbf{g}^{s}\right) \cdot \hat{\mathbf{S}}_{i}\right]
\end{array}
$$

where the three terms are respectively the antiferromagnetic Heisenberg interaction, the DM interaction, and the
Zeeman splitting energy. In addition to the superexchange coupling $J>0, \mathbf{D}$ is the $\mathrm{DM}$ vector, $\mathbf{H}$ the external field, and $\mathbf{g}^{u, s}$ are the uniform and staggered components of the gyromagnetic tensor. By making a local gauge transformation, which rotates the spins on two separate sublattices, and by neglecting all contributions at higher orders in $D / J$, Eq. (1) can be mapped to the simplified Hamiltonian

$$
\hat{H}=\sum_{i}\left[J \hat{\mathbf{S}}_{i} \cdot \hat{\mathbf{S}}_{i+1}-H S_{i}^{x}-(-1)^{i} h_{s} S_{i}^{z}\right]
$$

where $h_{s}$ is an effective staggered field proportional to the product of $H$ with a linear combination of $D$ and $g^{s}$; novel features therefore arise only when such a material is subject to an external magnetic field. The Hamiltonian of Eq. (2) can be mapped (by neglecting Zeeman splitting in the $z$-direction) into a sine-Gordon model, a minimal framework whose bosonized version provides a good description of the opening of the spin gap. $\underline{2-8}$ Further unconventional features of this model include the specific heat $\underline{\underline{6}}$, magnetic susceptibility,$\underline{\underline{3.9}}$ dynamical structure factor,,$\frac{5,7.8}{,}$ line shape in electron spin resonance (ESR) measurements,,$\underline{4}$ magnetization,,$\underline{9,10}$ and the presence of mid-gap states $11-14$

While these numerous studies assumed that the second term in Eq. (2) has no significant role for weak magnetic fields, in fact the uniform and staggered components may compete. A complete description of systems with DM interactions in arbitrary fields still requires the full exploration of the original Hamiltonian (1). A systematic investigation by Wang and coworkers 12 using the density-matrix renormalization-group (DMRG) tech- 
nique showed that the low-energy, high-field properties of copper benzoate are dominated by the uniform magnetic field, on which the spin gap depends linearly, while a crossover regime exists at intermediate fields. This prediction was later confirmed by ESR experiments in applied fields up to $35 \mathrm{~T}: \underline{15}$

Most staggered-field studies have focused on materials which are almost ideally $1 \mathrm{D}$ in nature, such as copper benzoate, $, 1,15-18$ copper pyrimidine, $, 10,19-21$

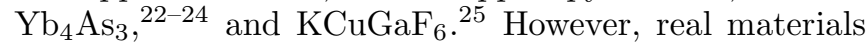
always have some interchain coupling, which in some cases may be comparable to the staggered magnetic field, if not also to the intrachain coupling. How the interchain interaction may change the essential physical properties remains an open question. Recent experiments on the weakly coupled chain system $\mathrm{CuCl}_{2} \cdot 2$ (dimethylsulfoxide) $(\mathrm{CDC})^{26,27}$ indicate that a gap opens at a finite value of $h_{s}$, rather than at zero, when the uniform magnetic field is applied; the power-law dependence of the excitation gap seems to be different from that observed in quasi1D materials. Early attempts to understand this behavior include calculations for the two-leg ladder $\frac{28,29}{,}$ which show a QPT taking place as a consequence of the competition between the staggered magnetic field and the interchain coupling. A chain mean-field theory developed ${ }^{30}$ to study the spin gap as function of the staggered field in $2 \mathrm{D}$ and 3D found a spin gap opening immediately with the applied field (the "noncompetitive case" defined in Sec. II and discussed in Sec. IV), a conclusion confirmed by DMRG $\underline{31}$

In a system where the staggered field competes with the ordering pattern favored by the magnetic interactions, a finite value of $h_{s}$ is required to induce a QPT. To date the only results available for this case are at the mean-field level, and may not deliver reliable conclusions for $2 \mathrm{D}$ systems. In particular, the linear dependence of the excitation gaps on the magnetic field appears to be inconsistent with experiment. ${ }^{26}$ In this paper we contribute to the understanding of coupled Heisenberg chains in staggered magnetic fields by performing continuous-time Monte Carlo simulations using the worm algorithm, $32-34$ and use our results to discuss the influence of interchain interactions and staggered fields on the ground state and the low-energy excitations.

This paper is organized as follows. In Sec. II we provide a formal introduction to the model and to the Monte Carlo method. In Sec. III we test our numerical techniques by computing the staggered magnetization in zero field, making contact with known results for coupledchain systems and the square lattice. In Sec. IV we present our Monte Carlo results for the noncompetitive case, which demonstrate the $2 \mathrm{D}$ nature of the system. Section V contains our complete results for the competitive case, including the determination of the QPT, the staggered magnetization in the ordered phase, the gap in the disordered phase with corresponding fitting exponents, and a comparison of the phase diagram with different mean-field theories. We summarize our investigation in Sec. VI.

\section{MODEL AND METHOD}

In this study we investigate the $S=1 / 2$ Heisenberg model on a spatially anisotropic square lattice in a staggered magnetic field. We stress that the purpose of our analysis is to determine, both qualitatively and quantitatively, the effects of a staggered field in different geometries. We do not aim to make a direct comparison with experiment, but rather to demonstrate the physical properties which may motivate the search for and characterization of an appropriate material in this class. Thus we focus here on effective models without the uniform magnetic field. For completeness we consider both the "noncompetitive" case, where the geometry of the magnetic interactions and the staggered field are commensurate, and the "competitive" case, where they are not. We consider an antiferromagnetic intrachain coupling with both ferromagnetic and antiferromagnetic interchain couplings, which we will label respectively as $(\pi, 0)$ and $(\pi, \pi)$. The two different spatial arrangements of the staggered field, also $(\pi, 0)$ and $(\pi, \pi)$, then give one competitive and one noncompetitive situation for each case.

The Hamiltonians can be expressed in the forms

$$
\begin{aligned}
\hat{H}_{1}= & \sum_{i, j=1}^{L} J \hat{S}_{i, j} \cdot \hat{S}_{i+1, j}+\sum_{i=1}^{L} J_{\perp} \hat{S}_{i, j} \cdot \hat{S}_{i, j+1} \\
& +\sum_{i=1}^{L}(-1)^{i+j} h_{s} S_{i, j}^{z} \\
\hat{H}_{2}= & \sum_{i=1}^{L} J \hat{S}_{i, j} \cdot \hat{S}_{i+1, j}+\sum_{i=1}^{L} J_{\perp} \hat{S}_{i, j} \cdot \hat{S}_{i, j+1} \\
& +\sum_{i=1}^{L}(-1)^{i} h_{s} S_{i, j}^{z}
\end{aligned}
$$

where $L$ is the linear dimension of the lattice and $J$ is the intrachain coupling, which we take as the unit of energy. The interchain coupling is $J_{\perp}, h_{s}$ is the magnitude of the effective staggered magnetic field, and $\hat{S}_{i, j}$ is the spin operator at lattice site $(i, j)$, with $i$ the index in the chain direction $(x)$ and $j$ the interchain index (y-direction). The four situations are represented schematically in Fig. 1 where the sign of $J_{\perp}$ determines the nature of the interchain interaction and the dots or crosses correspond to up and down orientations of the applied staggered field. Figures 1(a) and (b) represent respectively the non-competitive and competitive cases of model (3), while Figs. 1(c) and (d) represent the noncompetitive and competitive cases of model (4).

For our investigation of the ground states and lowest excitations of these models, we use the continuoustime worldline quantum Monte Carlo (QMC) method with "worm" updates. The concept of continuous-time 


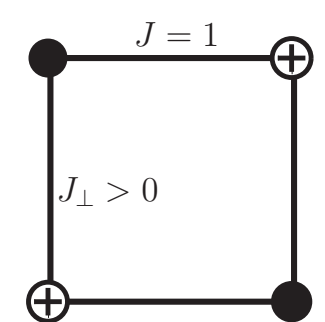

(a)

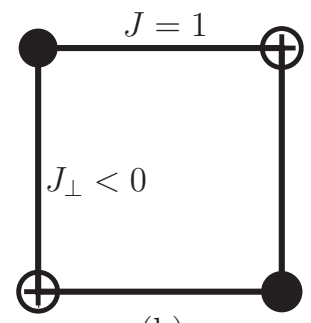

(b)

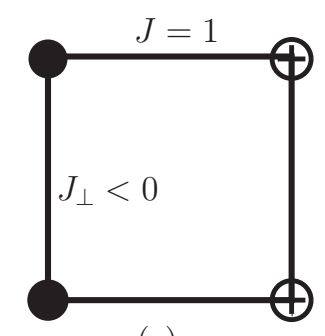

(c)

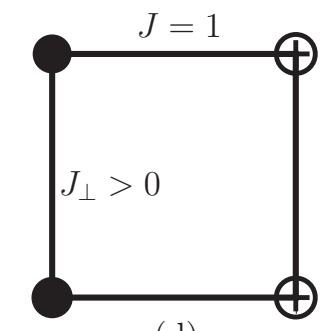

(d)
FIG. 1: Representation of couplings and staggered fields for the four possible cases considered here. Dots denote a field positive along $z$ and crosses a negative field. The magnetic interactions have $(\pi, \pi)$ geometry in cases (a) and (d), and $(\pi, 0)$ geometry in cases (b) and (c). The applied field geometry is $(\pi, \pi)$ in cases (a) and (b), which are described by Eq. (3), and $(\pi, 0)$ in cases (c) and (d) [Eq. (4)]. Cases (a) and (c) are therefore non-competitive, while (b) and (d) are competitive.

worldlines was first induced in QMC by N. V. Prokof'ev and coworkers in $1996 \stackrel{32}{\underline{2}}$ Unlike the standard, discretetime QMC algorithms, which are based on the SuzukiTrotter decomposition, in the continuous-time method the Hamiltonian $\hat{H}=\hat{H}_{0}+\hat{V}$ is separated into a diagonal term and a perturbation term. For a time-dependent perturbation, the partition function $Z=\operatorname{Tr}\left(e^{-\beta \hat{H}}\right)$ can be expressed as $Z=\operatorname{Tr}\left[e^{-\beta \hat{H}_{0}} U(\beta)\right]$, with $U(\beta)$ the Matsubara evolution operator,

$$
\begin{aligned}
U(\beta)= & 1-\int_{0}^{\beta} d \tau_{1} \hat{V}\left(\tau_{1}\right) U\left(\tau_{1}\right) \\
= & 1-\int_{0}^{\beta} d \tau_{1} \hat{V}\left(\tau_{1}\right) \\
& +\int_{0}^{\beta} d \tau_{1} \int_{0}^{\tau_{1}} d \tau_{2} \hat{V}\left(\tau_{1}\right) \hat{V}\left(\tau_{2}\right) U\left(\tau_{2}\right) \\
= & (-1)^{n} \sum_{n=0}^{\infty} \int_{0}^{\beta} d \tau_{1} \ldots \int_{0}^{\tau_{n-1}} d \tau_{n-1} \hat{V}\left(\tau_{1}\right) \ldots \hat{V}\left(\tau_{n}\right),
\end{aligned}
$$

in which $V(\tau)=e^{\tau H_{0}} V e^{-\tau H_{0}}$. In this series, each integral corresponds to a worldline configuration with $n$ "kinks," located at the points $0<\tau_{n}<\tau_{n-1}<\ldots<$ $\tau_{1}<\beta$ and varying continuously in the imaginarytime direction $\stackrel{32}{2}$ The integrals are evaluated by Monte
Carlo sampling of the kink configurations. In contrast to discrete-time QMC algorithms, there is no systematic error caused by imaginary-time discretization.

Based on this continuous-time worldline formulation, Prokof'ev and coauthors also developed an update algorithm based on breaking a worldline by inserting a pair of creation and annihilation operators, which then evolve by local moves $\frac{33}{3}$ (This became known as the "worm" update from the motion of pairs of worldline kinks.) The algorithm considers two configuration spaces, denoted Z and $\mathrm{G}$ : while $\mathrm{Z}$ contains only closed worldlines, and is the space of the partition function, $\mathrm{G}$ contains a worldline broken by a physical process connecting points $\left(\vec{r}_{i}, \tau_{i}\right)$ and $\left(\vec{r}_{j}, \tau_{j}\right)$. The $\mathrm{Z}$ and $\mathrm{G}$ configuration spaces can be exchanged by creation or annihilation of an $(i, j)$ "worm" pair on the same flat worldline. This is equivalent to processes in the grand canonical ensemble. In the $\mathrm{G}$ configuration space, processes which move the worms in both time (changing worm position in the imaginary time direction on the same site) and space (the worm is moved to a neighboring site, creating or annihilating a kink) update the configuration and change its structure.

In the $\mathrm{Z}$ configuration space it is easy to compute thermodynamic quantities such as energy, magnetization, susceptibility, and specific heat. In the $\mathrm{G}$ configuration space, each accepted worm move results in a contribution to the histogram of the Green function, $G\left(\vec{r}_{i}-\vec{r}_{j}, \tau_{i}-\tau_{j}\right)$. With sufficiently many Monte Carlo steps, one obtains a convergent Matsubara Green function, defined as

$$
G(r, \tau)=G\left(\vec{r}_{i}-\vec{r}_{j}, \tau_{i}-\tau_{j}\right)=\left\langle\mathcal{T}_{\tau} S_{\vec{r}_{i}}^{+}\left(\tau_{i}\right) S_{\vec{r}_{j}}^{-}\left(\tau_{j}\right)\right\rangle
$$

where $\mathcal{T}_{\tau}$ is is the time-ordering operator. The average $\langle\ldots\rangle$ is obtained from the histogram, and is normalized by $G(0,0)$. From the statistical average of the Green function, one may obtain the critical exponents of $G$, the single-particle excitation spectrum, and certain correlation functions.

In comparison with previous results by the DMRG technique, $\stackrel{31}{=} \mathrm{QMC}$ algorithms have the major advantage of treating $2 \mathrm{D}$ models. In the continuous-time worldline approach, lattice sizes of $L$ up to 100 and inverse temperatures $\beta=1 / T$ up to 100 can be accommodated in approximately 24 hours of calculation time when working on a server with an Inter XEON E5460 CPU. A further advantage of QMC is that it is easy to work with periodic boundary conditions, so that there are no difficulties caused by edge states. ${ }^{31}$ We therefore expect that reliable numerical results can be obtained for the $2 \mathrm{D}$ case by this technique. In this paper, we focus on the energy gap $\Delta$, which characterizes gapped phases, and the transverse staggered magnetization $M_{\perp}^{s}$, which is characteristic of ordered phases. The gap is obtained ${ }^{34}$ from the Green function through the expression

$$
\Delta=-\frac{\ln \left[G(p, \tau) / G\left(p, \tau_{0}\right)\right]}{\tau-\tau_{0}},
$$

while the staggered correlation function

$$
C_{\mathbf{Q}}^{s}(r)=\left\langle\hat{S}_{0}^{+} \hat{S}_{\mathbf{r}}^{-} e^{i \mathbf{Q} \cdot \mathbf{r}}\right\rangle
$$




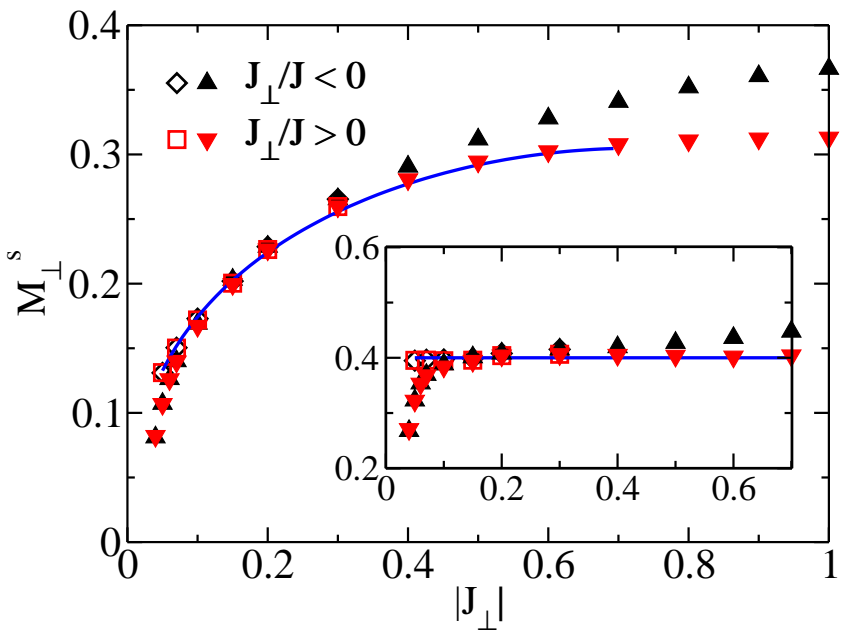

FIG. 2: (Color online) Staggered magnetization $M_{\perp}^{s}$ as a function of interchain coupling for both signs of $J_{\perp}$, at zero staggered field. The isotropic square lattice is represented by $J_{\perp}=1$. Inset: $M_{\perp}^{s}$ as a function of $-\sqrt{\left|J_{\perp}\right|}(1+$ $\left.0.095\left|J_{\perp}\right|\right) \ln ^{1 / 3}\left|J_{\perp} / 1.3\right|,{ }^{37}$ showing the strong logarithmic corrections to the square-root form of a chain mean-field theory. Up- and down-pointing triangles denote data obtained for $L \times L$ samples with $L=100$, while squares and diamonds denote data obtained on rectangular samples with aspect ratio $L_{x} / L_{y}=8$. The solid line is the fitting function deduced in Ref. 37.

is obtained from the Green-function histogram. The staggered magnetization is defined as $M_{\perp}^{s}=\sqrt{\sum_{\mathbf{r}} C_{\mathbf{Q}}^{s}(r)} / N$, where $\mathbf{Q}=(\pi, \pi)$ for model (3), and $\mathbf{Q}=(\pi, 0)$ for model (4).

\section{MAGNETIZATION AT ZERO FIELD}

We begin by investigating the case $h_{s}=0$, which also serves to benchmark our method for accuracy and systematic errors. We consider both the spatially anisotropic antiferromagnetic Heisenberg square lattice and the lattice of Heisenberg chains with ferromagnetic interchain coupling. For $\left|J_{\perp}\right| / J>0.15$, the lattice size $L$ in our calculation is 100 , with $\beta$ set equal to $L$. For lower values of the interchain coupling, we find that rectangular lattices are required to achieve reliable results, and we adjust the cluster aspect ratio accordingly. The staggered magnetization we compute for the $2 \mathrm{D}$ antiferromagnetic Heisenberg model is shown in Fig. 2, For benchmarking purposes, in the isotropic square lattice $\left(J=J_{\perp}=1\right)$ we find $M_{\perp}^{s}=0.313 \pm 0.006$, which agrees very well with the known value of $0.306 \stackrel{40}{0}$ The case of ferromagnetic interchain coupling is not entirely symmetrical, the nonuniversal behavior at larger values of $\left|J_{\perp}\right|$ being manifest as a more rapid saturation in the antiferromagnetic case.

The problem of the weakly coupled Heisenberg spin chains is a fundamental one in quantum magnetism, and has received a great deal of attention over the last five decades. It encapsulates the physics of the crossover from truly 1D systems, dominated by quantum fluctuation effects, to high-dimensional, renormalized classical behavior. Because the $S=1 / 2$ Heisenberg chain is already critical, with a gapless ground state, any transverse coupling in an unfrustrated geometry is thought to give rise to magnetic order, and extensive investigation has reinforced the general agreement that the critical $J_{\perp c}=0$. Early discussion of the critical behavior of the ordered moment and Néel temperature used the highdimensional framework of renormalized spin waves, and suggested a purely logarithmic rise of $M_{\perp}$ with $\left|J_{\perp}\right|, \frac{35}{36}$ By contrast, in a chain-based weak-coupling approach,, 36 where the transverse interactions are modeled as an effective staggered magnetic field, the ordered moment has a power-law dependence, $M_{\perp} \propto \sqrt{\left|J_{\perp}\right|}$, albeit with suspected logarithmic corrections. The definitive study of the problem was performed by Sandvik, $\stackrel{37}{,}$ using QMC within a multichain mean-field theory, and reveals strong logarithmic corrections to the square-root dependence, $M_{\perp} \propto-\sqrt{\left|J_{\perp}\right|} \ln ^{1 / 3}\left|J_{\perp}\right|$ (with a weak additive linear term). The power of $1 / 3$ in the logarithm was also found in the problem of the single chain in a staggered field. ${ }^{3}$ The importance of the logarithmic terms lies in the presence of marginally irrelevant operators, which are neglected in the transformation of the high-dimensional Heisenberg model to a chain in an effective staggered field.

Our data for both signs of the transverse coupling, shown in Fig. 2, have the same form at small $\left|J_{\perp}\right|$. Our results are fully consistent with those of Ref. [37]. First, we confirm our sensitivity to the expected logarithmic corrections, which will be essential in the sections to follow. Second, our results for square lattices deviate from the expected form at values of the transverse coupling below $\left|J_{\perp}\right| \approx 0.15$. We simulate instead rectangular systems of different aspect ratios up to $L_{x} / L_{y}=8, \frac{37}{3}$ which allows us to obtain accurate values of $M_{\perp}$ at least to $\left|J_{\perp}\right| / J=0.05$, below which the calculations become very time-consuming. This study of the staggered magnetization at $h_{s}=0$ therefore allows us to benchmark the accuracy of our results for highly anisotropic systems. The effect of the finite temperature in our calculations is not thought to be significant $\frac{37}{2}$ Quantifying the logarithmic corrections to the chain mean-field picture in this way will be important in Sec. V, where we will use it as a guide to understanding our numerical results in the presence of a staggered field. We will show that the field acts to alter significantly the effective dimensionality of the system and the relevance of these logarithmic terms.

\section{NONCOMPETITIVE CASE}

In the two noncompetitive cases, the magnetic interactions and the applied staggered field have the same spatial pattern [Figs. 1(a) and (c)]. For any finite value of $J_{\perp}$, the system is magnetically ordered at $h_{s}=0$, by 


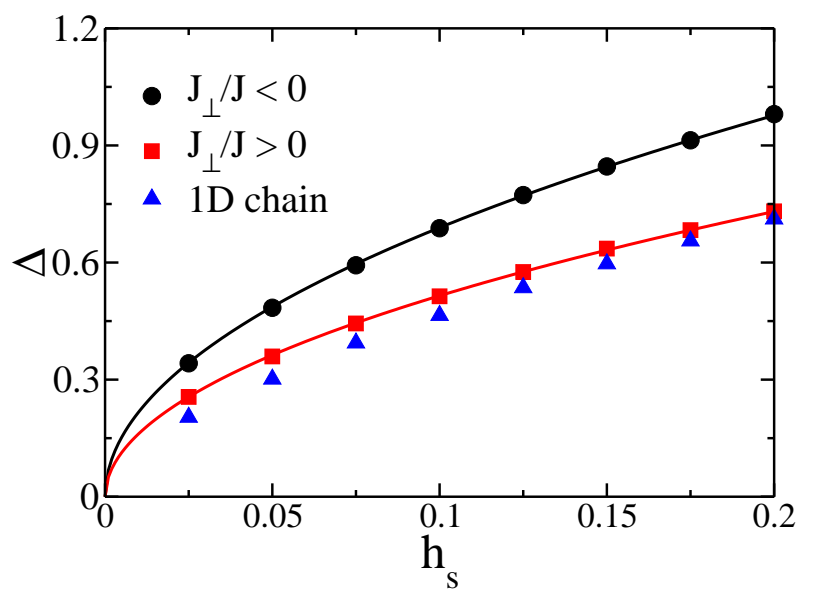

FIG. 3: (Color online) Excitation gap as a function of staggered magnetic field for the $2 \mathrm{D}$ spin- $\frac{1}{2}$ Heisenberg square lattice $\left(J_{\perp}=J\right)$ in the noncompetitive case. Monte Carlo results are presented as filled circles for the $(\pi, \pi)$ geometry [model (3)] and as squares for $(\pi, 0)$ [model (4)]. The solid lines are fitting curves. Shown also (triangles) are Monte Carlo results for the Heisenberg chain.

a spontaneous breaking of the $\mathrm{SU}(2)$ spin symmetry, and has massless spin-wave excitations. When a commensurate staggered field is applied, the symmetry is broken explicitly as the field direction selects the spin orientation. As for a ferromagnet in a uniform field, the staggered field serves as an anisotropy term, which opens a spin-wave gap for any finite value of $h_{s}$. In the conventional mean-field approach,, 30 a standard linear spin-wave approximation gives a gap

$$
\Delta \approx \sqrt{4 S J h_{s}\left[1+\left(h_{s} / 4 S J\right)\right]}
$$

By extrapolation from smooth and rapidly convergent DMRG results for antiferromagnetic Heisenberg ladders in staggered fields, the authors of Ref. 31] also obtained a $2 \mathrm{D}$ field-dependence of $\Delta=(2.27 \pm 0.01) h_{s}^{0.50 \pm 0.01}$. However, the noncompetitive geometry may also be discussed within a chain mean-field theory, where the interchain interactions are treated as an effective staggered

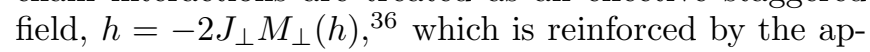
plied staggered field. In this treatment, the dominant physics is the breaking of the continuous symmetry of the spin direction, and the exact dependence on $J_{\perp}$ is not clear; that the gap $\Delta$ is independent of $J_{\perp}$ in Eq. (9) underlines the fact that it is in essence purely an effect of the field on the chain, which would suggest some influence of $1 \mathrm{D}$ physics.

Our analysis is the first direct numerical calculation of the gap evolution in this case. We begin by considering the "isotropic" square lattice $\left(\left|J_{\perp}\right|=J\right)$ with systems of $L=100$ and temperatures $\beta=100$, to maximize the reliability of our data by systematic extrapolation. In Fig. 3 we show the dependence of the gap on the staggered field for both noncompetitive cases. A fit to the formula

$$
\Delta=a_{0} h_{s}^{\alpha}
$$

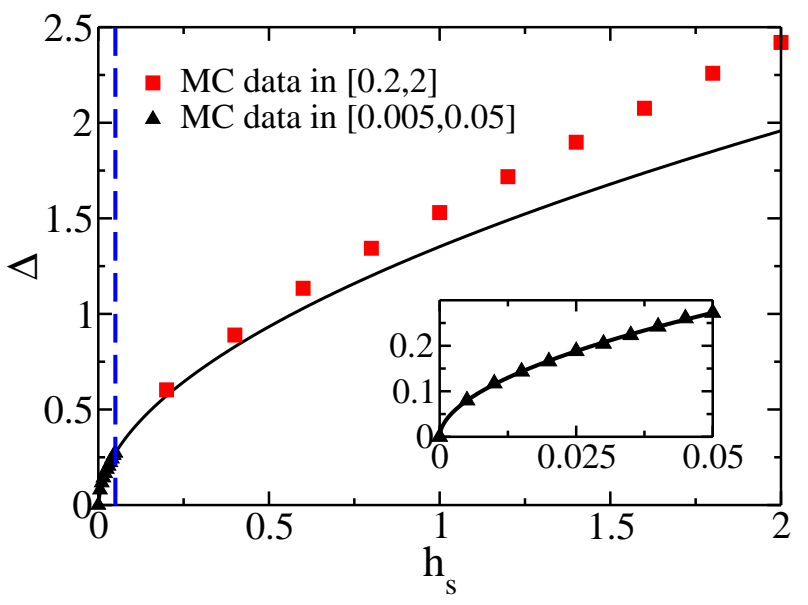

FIG. 4: (Color online) Excitation gap as a function of staggered field for the anisotropic $2 \mathrm{D}$ spin- $\frac{1}{2}$ antiferromagnetic Heisenberg model in the noncompetitive case for $J_{\perp} / J=0.1$. Triangles and squares correspond respectively to $h_{s}$ steps of 0.005 and 0.2 . The fitting curve is obtained from the data with $\delta h_{s}=0.005$ (inset). The blue, dashed line marks the 2D regime for $J_{\perp} / J=0.1$.

yields excellent results (Fig. 3), with $\Delta=2.19 h_{s}^{0.50 \pm 0.01}$ for the $(\pi, \pi)$ case [Fig. 1(a)] and $\Delta=1.65 h_{s}^{0.50 \pm 0.01}$ for the $(\pi, 0)$ case [Fig. $1(\mathrm{c})]$. The exponents in both cases agree perfectly with the result predicted both by meanfield theory and by extraolation from Heisenberg ladders. The isotropic square lattice shows unambiguously $2 \mathrm{D}$ behavior, with an immediate opening and square-root growth of the gap.

By contrast, in the purely $1 \mathrm{D}$ case is it known ${ }^{2,3}$ that the gap opens according to $\Delta \propto h_{s}^{2 / 3}$ with logarithmic corrections. This behavior is shown as the triangles in Fig. 3. The more rapid growth of the gap in 2D may be considered heuristically as the consequence of a mutual reinforcement of the applied and effective staggered fields, the latter arising from the magnetic moment enhanced by the former. The key question to address is whether the system displays any kind of continuous crossover, as a function of $\left|J_{\perp}\right|$, from a regime characterized by $1 \mathrm{D}$ exponents to a $2 \mathrm{D}$ regime. By taking the non-competitive $(\pi, \pi)$ case at $J_{\perp}=0.1$ and fitting the

TABLE I: Fitting coefficients and exponents for five data sets with different point spacings $\delta h_{s}$.

\begin{tabular}{c|c|c}
\hline \hline \multicolumn{3}{c}{$J_{\perp} / J=0.1$} \\
\hline$\delta h_{s}$ & $a_{0}$ & $\alpha$ \\
\hline 0.005 & 1.351 & 0.535 \\
0.01 & 1.415 & 0.548 \\
0.05 & 1.512 & 0.574 \\
0.1 & 1.522 & 0.583 \\
0.2 & 1.554 & 0.624 \\
\hline \hline
\end{tabular}



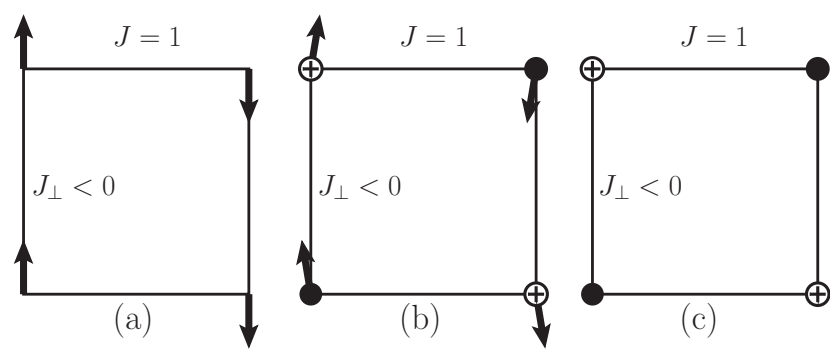

FIG. 5: Schematic representation of the spin state in the competitive case on varying the staggered field $h_{s}$. The system has FM interchain interactions and a $(\pi, \pi)$ staggered field [model (3)]. Black arrows represent the spin directions and the staggered field is represented by dots (up) and crossed circles (down).

gap obtained from ten sets of data points with five different $h_{s}$ intervals, $0.005,0.01,0.05,0.1$, and 0.2 , we find the fitting parameters listed in Table प. It is clear that the exponent changes from a $2 \mathrm{D}$ form to a $1 \mathrm{D}$ form: for large $h_{s}$, the system is effectively no longer aware of the coupling, and has 1D behavior, while at sufficiently small $h_{s}$ the $2 \mathrm{D}$ behavior always emerges. The same result is illustrated in Fig. 4using the data points with $\delta h_{s}=0.005$ and $\delta h_{s}=0.2$. It is clear that the latter data set does not fall on the square-root curve obtained from the former. We may conclude that the $2 \mathrm{D}$ regime in this model is given approximately by $h_{s}<J_{\perp} / 2$.

\section{COMPETITIVE CASE}

We turn now to the competitive case, where the interchain coupling and the staggered field compete to establish the pattern of magnetic order. From a meanfield analysis of this system, $\underline{\underline{30}}$ there exist two different phases at finite $h_{s}$, an ordered phase with spontaneous symmetry-breaking (SSB, of the continuous U(1) symmetry) in the plane normal to the staggered field and a gapped, "symmetric" phase in which the spins are oriented in the field direction.

A phenomenological description of the situation is illustrated in Fig. 5 for the case of a $(\pi, \pi)$ staggered field applied to a system with $J_{\perp}<0$. At $h_{s}=0$, the system is ordered with a spontaneous breaking of $\mathrm{SU}(2)$ symmetry and one-site translational invariance in the transverse direction. For $h_{s}=0^{+}$, a spin-flop transition occurs into the plane perpendicular to the field, represented as the $(x y)$ plane, but there remains a SSB in this plane [Fig. [5(a)]. For finite $h_{s}$, the spins are forced to rotate into the direction of the staggered field, or into the $y z$ plane in Fig. 5(b), adopting a canted structure with twosite translational invariance in the transverse direction. The finite magnetic order parameter in this phase is reduced by the staggered field, and the excitations of the system remain gapless. Finally, when the staggered field is increased beyond a critical value $h_{c}$, the order param-

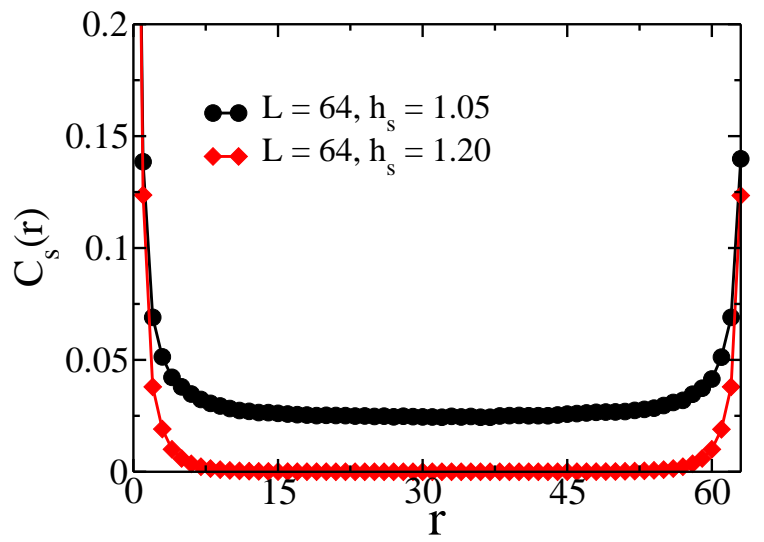

FIG. 6: (Color online) Staggered correlation function $C_{s}(r)=$ $\left\langle\hat{S}_{0}^{+} \hat{S}_{\mathbf{r}}^{-} e^{i \mathbf{Q} \cdot \mathbf{r}}\right\rangle$ (see text) with $L=64$ and $J_{\perp} / J=-1.0$, for two staggered fields, $h_{s}=1.05$ and 1.2 , chosen to represent respectively the ordered and the gapped phase.

eter is driven to zero, long-range order collapses, and the spins are fully oriented in the field direction [Fig. [5 (c)].

We begin by our numerical analysis by illustrating the staggered correlation function within the system. Figure 6 shows $C_{s}(r)$ as a function of distance for model (3), with $J_{\perp}=-1$ and for two values of $h_{s}$. For $h_{s}=1.05$, staggered correlations are finite and there is long-range order in the plane transverse to the applied field. By contrast, for $h_{s}=1.2, C_{s}(r)$ falls to zero abruptly away from the edges of the system; these two values of $h_{s}$ therefore fall on opposite sides of the anticipated critical staggered field, $h_{c}$. We use the staggered magnetization to analyze both the SSB phase and the field-induced quantum phase transition between the SSB and symmetric phases. We characterize the symmetric phase by its gap, defined in Eq. (7), and by the scaling of this gap.

\section{A. Critical point}

From the discussion above, the critical point $h_{c}$ is the single most important quantity in the descriptiontion of the competitive case. Once $h_{c}$ is determined, a process achieved most accurately using the behavior of the order parameter in the SSB phase, the gap and the 2D excitations of the symmetric phase can be calculated with high accuracy. To determine the critical point, or the phase boundary in the space of $J_{\perp}$ and $h_{s}$, we apply the finitesize-scaling hypothesis to the behavior of the staggered correlation functions $C_{\pi, 0}$ and $C_{\pi, \pi}$ [respectively for models (3) and (4)] as functions of $L$. These are expected ${ }^{39}$ to obey the scaling form

$$
C(\delta)=L^{2-d-z} f\left(\delta L^{1 / \nu}, \beta / L^{z}\right),
$$

where $\delta=h_{s}-h_{c}, \beta$ is the inverse temperature, and $z$ is the dynamical exponent.

The critical point $h_{c}$ can be measured accurately by computing the staggered correlation function near the 

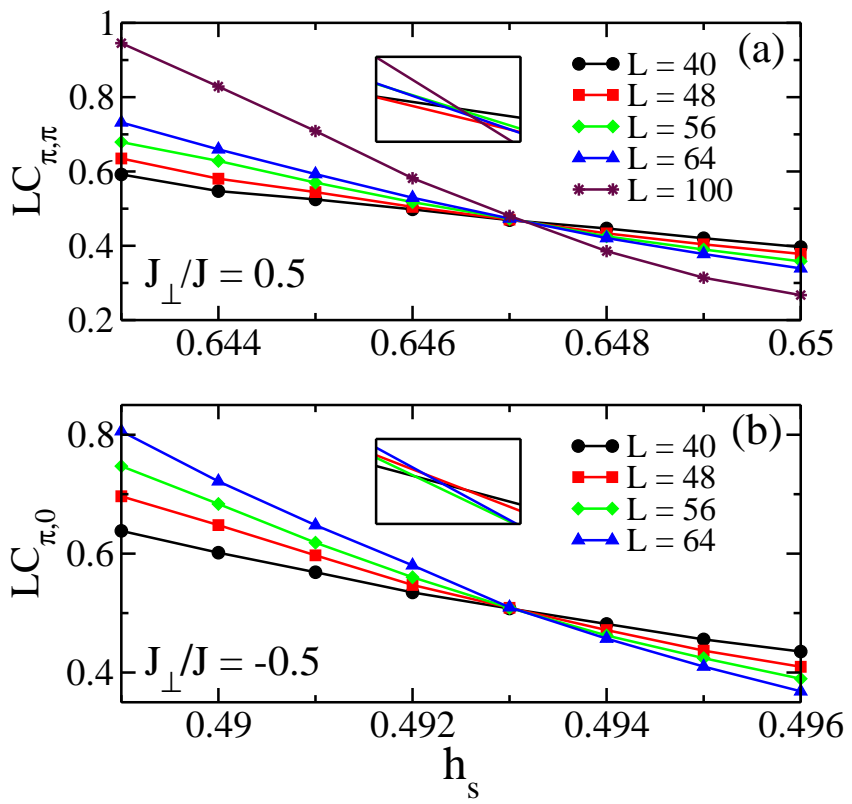

FIG. 7: (Color online) Determination of $h_{c}$ from the finitesize-scaling hypothesis for (a) $J_{\perp}=0.5$ and (b) $J_{\perp}=-0.5$. Error bars are set by the inexact crossing of the lines, shown in the insets. In panel (a), $0.6471 \leq h_{s} \leq 0.6472$ and $0.462 \leq$ $L C_{\pi, \pi} \leq 0.471$. In panel (b), $0.49300 \leq h_{s} \leq 0.49315$ and $0.502 \leq L C_{\pi, 0} \leq 0.510$.

critical point for different lattice sizes $L \times L$. On fixing $\beta / L^{z}=1$, the scaling function $f$ depends on a single parameter, $\delta L^{1 / \nu}$. Precisely at the critical point, $\delta=0$, $C L^{d+z-2}$ is independent of the lattice size, which implies that calculated curves for $C L^{d+z-2}$ as functions of $h_{s}$ for different lattice sizes should cross at the critical point. It is clear from this form that $z$ is a measure of the system coherence: as $z$ becomes larger, the coherence of the system vanishes more rapidly in space at the critical point. Here we test that the dynamical exponent takes the value $z=1$, and we find this to hold at all points around the critical region.

We have simulated 11 different values of $\left|J_{\perp}\right|$ for both ferro- and antiferromagnetic interchain coupling, with fixed $L / \beta=1$ and at least four lattice sizes, namely $L=40,48,60$, and 64 . We have verified the accuracy of our results by performing calculations with $L=100$ for the case $J_{\perp}=0.5$. For a determination of the critical point, we work only with square samples at all values of $\left|J_{\perp}\right|$; the simultaneous vanishing of the superfluid density (spin stffness) in both directions at $h_{c}$ negates the advantanges of rectangular samples for low $\left|J_{\perp}\right|$ (Sec. III) in this case. Examples of the sets of crossing curves are illustrated in Fig. 7 for $J_{\perp}=0.5$ and -0.5 . The error in $h_{c}$ can be estimated from the separation of the different intersection points within a single manifold of curves; all errors $\delta h_{c}$ are of order $10^{-4}$. The full numerical details determined from this procedure are presented in Table III The phase diagram deduced from these values of $h_{s}$ is discussed in Sec. VD.

\section{B. Staggered Magnetization}

For applied fields $h_{s}<h_{c}$, the SSB phase possesses a transverse staggered moment (or off-diagonal long-range order) $M_{\perp}^{s}$. We have calculated this quantity for the case $h_{s}=0$ in Sec. III. In general one expects the magnetic order to be suppressed by the competing staggered field, because of the different ordering patterns they favor, until $M_{\perp}^{s}$ vanishes at $h_{s}=h_{c}$. In Fig. 8 we show $M_{\perp}^{s}$ as a function of $h_{s}$ for different values of the interchain coupling $J_{\perp}$. For larger values of $J_{\perp}$, of both signs, the order parameter shows a conventional, monotonic decrease [Fig. 8(a)]. However, when $\left|J_{\perp}\right| \lesssim 0.2$, for both signs of $J_{\perp}$ [Figs. $8(\mathrm{~b})$ and (c)], we observe that $M_{\perp}^{s}$ first increases despite the increase in the competing staggered field. We stress that our results for small $\left|J_{\perp}\right|$ were obtained on rectangular samples with aspect ratio $L_{x} / L_{y}=8$, following the procedure established in Sec. III as providing the highest available accuracy.

To our knowledge, this novel and purely quantum mechanical effect has not been remarked upon previously in coupled Heisenberg chains. An analogous effect can be found in dimerized quantum magnets 41 such as $\mathrm{NH}_{4} \mathrm{CuCl}_{3}$, where an applied uniform magnetic field, which in principle competes with the transverse staggered order, nonetheless causes the staggered moment to rise at the same time as the uniform polarization is increased. A heuristic understanding of this phenomenon is that all forms of magnetic order are in fact competing with quantum fluctuation effects favoring disordered states. When the disordered state is suppressed by an applied field, more ordered spin "weight" is available both for the magnetization component favored by the field and for the component favored by the magnetic interactions.

As $h_{s}$ is increased further, $M_{\perp}^{s}$ is suppressed for all

TABLE II: Critical staggered fields $h_{c}$ for systems with transverse couplings $\left|J_{\perp}\right|$ varying from 0.05 to 1 . Shown are results for both ferromagnetic interchain couling in a $(\pi, \pi)$ staggered field (3) and antiferromagnetic interchain coupling in a $(\pi, 0)$ field.

\begin{tabular}{c|c|c|c|c|c}
\hline \hline \multicolumn{3}{c|}{$(\pi, \pi)$} & \multicolumn{3}{c}{$(\pi, 0)$} \\
\hline$J_{\perp}$ & $h_{c}$ & $\delta h_{c}$ & $J_{\perp}$ & $h_{c}$ & $\delta h_{c}$ \\
\hline-0.05 & 0.02459 & 0.00007 & 0.05 & 0.02606 & 0.00008 \\
-0.1 & 0.06336 & 0.00011 & 0.1 & 0.06964 & 0.00027 \\
-0.2 & 0.15805 & 0.00015 & 0.2 & 0.18495 & 0.00013 \\
-0.3 & 0.26495 & 0.00016 & 0.3 & 0.32470 & 0.00022 \\
-0.4 & 0.37775 & 0.00014 & 0.4 & 0.48034 & 0.00008 \\
-0.5 & 0.49305 & 0.00012 & 0.5 & 0.64715 & 0.00015 \\
-0.6 & 0.60990 & 0.00006 & 0.6 & 0.82166 & 0.00018 \\
-0.7 & 0.72630 & 0.00012 & 0.7 & 1.00225 & 0.00020 \\
-0.8 & 0.84300 & 0.00011 & 0.8 & 1.18617 & 0.00014 \\
-0.9 & 0.95850 & 0.00017 & 0.9 & 1.37339 & 0.00015 \\
-1.0 & 1.07300 & 0.00021 & 1.0 & 1.56290 & 0.00019 \\
\hline \hline
\end{tabular}



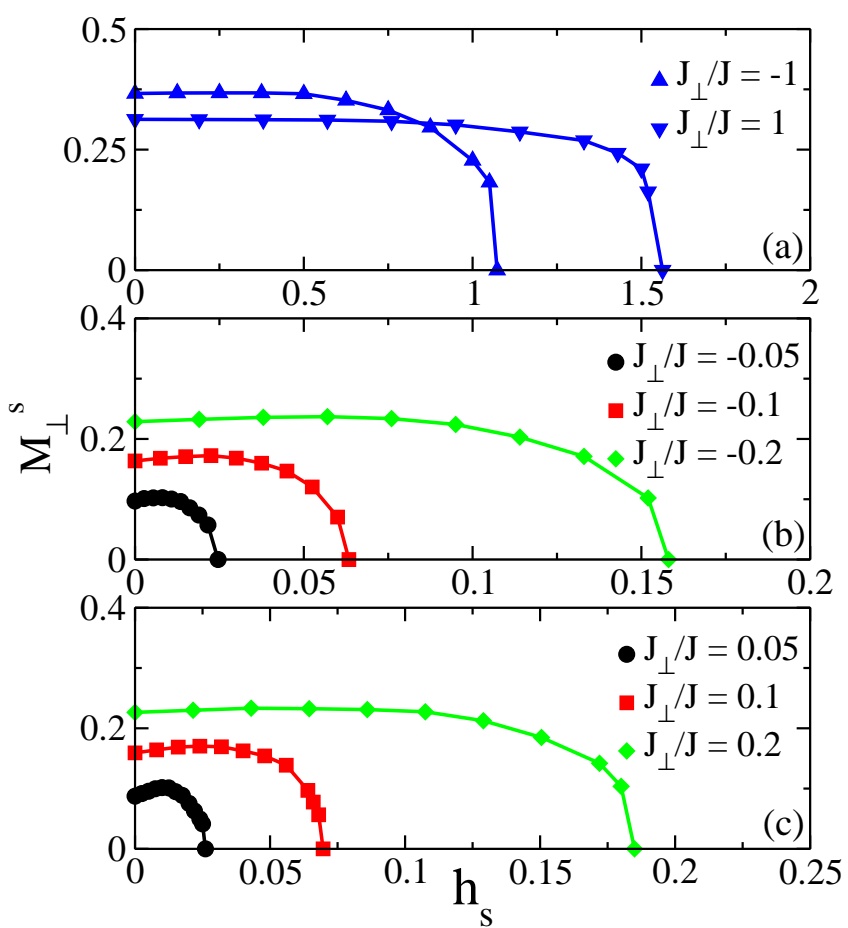

FIG. 8: (Color online) Transverse staggered order parameter $M_{\perp}^{s}$ as a function of $h_{s}$ for different values of the interchain coupling $J_{\perp}$. (a) Regime of large $\left|J_{\perp}\right|$, showing conventional suppression of $M_{\perp}^{s}$ by the competing field $h_{s}$. (b) and (c) Regime of small $\left|J_{\perp}\right|$, showing unconventional increase of $M_{\perp}^{s}$ with $h_{s}$ at low applied fields.

values of $\left|J_{\perp}\right|$, and it falls continuously to zero at the second-order quantum phase transition. In this regime the finite-size effects in our simulations are large, even though the statistical errors are very small. Unlike the determination of $h_{c}$ itself, there is neither a systematic approach by extrapolation with different system sizes, which may allow a sufficiently accurate determination of $M_{\perp}^{s}$ close to $h_{c}$, nor a method for the accurate estimation of such errors. Thus we are not able to deduce the critical exponents of the transverse staggered moment in order to characterize this side of the quantum phase transition. The results of Sec. VC below suggest that some anomalous behavior may be expected.

\section{Energy gap}

In the gapped, symmetric phase we wish to consider the properties of the excited states. We compute the energy gap $\Delta$ and extract the scaling behavior of the gap in the region $h_{s}-h_{c}<\left|J_{\perp}\right| / 2$ deduced in Sec. IV. In Fig. 9 we present selected examples of the function $\Delta\left(h_{s}\right)$ for a range of positive and negative values of $J_{\perp}$. Unlike in the SSB phase, there are no strong finite-size effects on the gap data close to $h_{c}$ for the symmetric phase, a result we have confirmed by studies on lattices of various sizes up to $L=100$. For a given $h_{c}$, determined from
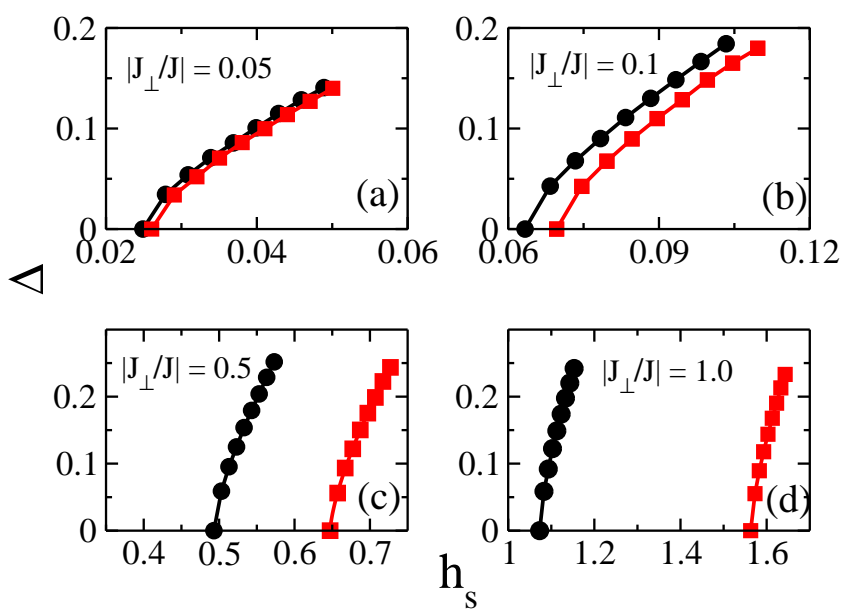

FIG. 9: (Color online) Gaps as a function of staggered magnetic field $h_{s}$ for four different values of $\left|J_{\perp}\right|$. Monte Carlo results for the gap are presented by black circles and red squares respectively for ferro- and antiferromagnetic interchain couplings. The critical points $h_{c}$ are determined from finite-size scaling of the transverse order parameter in the SSB phase. The gap is fitted to the form $\Delta=a_{0}\left(h_{s}-h_{c}\right)^{\alpha}$. Fitting curves are shown by solid lines and complete fitting parameters presented in Table III.

finite-size scaling of the transverse order parameter in the SSB phase, we collect eight data points within the scaling regime $h_{s}-h_{c}<\left|J_{\perp}\right| / 2$. We fit our numerical data to the formula $\Delta=a_{0}\left(h_{s}-h_{c}\right)^{\alpha}$.

Our gap calculations are of course consistent with the values of $h_{c}$ computed in the SSB phase. The gaps for $J_{\perp}$ values of opposite sign show only small, quantitative differences. The two gaps converge to that of the $1 \mathrm{D}$ chain when $J_{\perp} \rightarrow 0$. As in Sec. VA, $h_{c}$ increases with the mag-

TABLE III: Fitting coefficients and exponents for the excitation gap in systems with traverse couplings $\left|J_{\perp}\right|$ varying from 0.05 to 1 . Shown are results for both ferromagnetic interchain coupling in a $(\pi, \pi)$ staggered field (3) and antiferromagnetic interchain coupling in a $(\pi, 0)$ field (4).

\begin{tabular}{c|c|c|c||c|c|c|c}
\hline \hline \multicolumn{7}{c|}{$(\pi, \pi)$} & \multicolumn{4}{|c}{$(\pi, 0)$} \\
\hline$J_{\perp}$ & $a_{0}$ & $\alpha$ & $\delta \alpha$ & $J_{\perp}$ & $a_{0}$ & $\alpha$ & $\delta \alpha$ \\
\hline-0.05 & 0.929 & 0.693 & 0.003 & 0.05 & 0.943 & 0.698 & 0.004 \\
-0.1 & 0.926 & 0.718 & 0.005 & 0.1 & 0.880 & 0.708 & 0.010 \\
-0.2 & 0.862 & 0.724 & 0.006 & 0.2 & 0.822 & 0.715 & 0.005 \\
-0.3 & 0.815 & 0.718 & 0.006 & 0.3 & 0.759 & 0.706 & 0.008 \\
-0.4 & 0.761 & 0.704 & 0.004 & 0.4 & 0.744 & 0.703 & 0.003 \\
-0.5 & 0.735 & 0.700 & 0.004 & 0.5 & 0.732 & 0.702 & 0.006 \\
-0.6 & 0.716 & 0.693 & 0.003 & 0.6 & 0.686 & 0.692 & 0.008 \\
-0.7 & 0.709 & 0.694 & 0.005 & 0.7 & 0.676 & 0.689 & 0.009 \\
-0.8 & 0.705 & 0.692 & 0.005 & 0.8 & 0.673 & 0.688 & 0.005 \\
-0.9 & 0.698 & 0.692 & 0.007 & 0.9 & 0.667 & 0.686 & 0.006 \\
-1.0 & 0.693 & 0.692 & 0.007 & 1.0 & 0.661 & 0.686 & 0.008 \\
\hline \hline
\end{tabular}




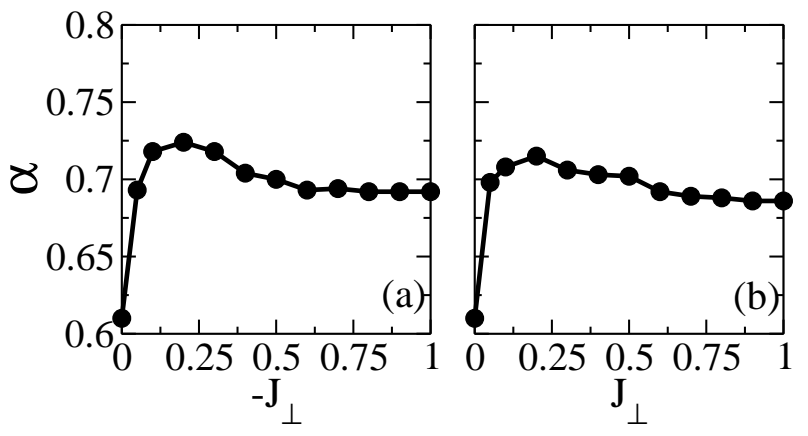

FIG. 10: (Color online) Critical exponents $\alpha$ of the gap $\Delta$ (Table III) as a function of $\left|J_{\perp}\right|$ for (a) ferromagnetic interchain coupling and (b) antiferromagnetic interchain coupling. The solid lines are a guide to the eye.

nitude of $J_{\perp}$, reflecting the fact that it is the competition between the staggered field and the interchain coupling that drives the quantum phase transition. Full details of the fitting parameters for the gap, including the errors in the exponents we extract, are listed in Table III.

Figure 10 shows the gap exponent extracted for different values of $J_{\perp}$. Because all of the data is fitted to a simple power law, the logarithmic corrections in the $1 \mathrm{D}$ chain result in an effective exponent of 0.61 for $J_{\perp}=0$. The exponent shows an initial increase as $\left|J_{\perp}\right|$ increases, but then falls weakly and remains close to $\alpha=0.7$ over the remainder of the range up to $\left|J_{\perp}\right|=1$. The values for the two cases of ferro- and antiferromagnetic $J_{\perp}$ differ only very slightly, and no universal value is indicated. It is clear, however, that the value of $\alpha$ does not fall to 0.5 , even arbitraily close to the transition, as would be expected in a $2 \mathrm{D}$ mean-field theory or from the results of Sec. IV. Our results therefore provide strong evidence for nontrivial quantum physics.

For a heuristic explanation of these data, we appeal to the chain mean-field theory of Ref. [36], where the interchain interactions are treated as an effective staggered field $h_{0}=-2 J_{\perp} M_{\perp}^{0}$. The presence of a real staggered field either reinforces this effective one, as in the noncompetitive geometry of Sec. IV, or suppresses it in the competitive geometry. Unlike the noncompetitive case, where the applied field breaks all continuous symmetries, in the competitive case a $\mathrm{U}(1)$ symmetry is maintained and the system may still be treated as a chain in a single effective field.

At lowest order, one may write $h_{\text {eff }}=-2 J_{\perp} M_{\perp}\left(h_{s}\right)=$ $-2 J_{\perp} M_{\perp}^{0}+h_{s}$, from which $M_{\perp}\left(h_{s}\right)=M_{\perp}^{0}(1-$ $\left.h_{s} / 2 J_{\perp} M_{\perp}^{0}\right)$. This simple linear approximation contains directly the competition between the applied and effective staggered fields, and suggests a quantum phase transition at $h_{c}=2 J_{\perp} M_{\perp}^{0}$, where the applied field cancels the interactions. The quasi-linear relation between $h_{c}$ and $J_{\perp}$ is clear from our calculations, while the relevance of next-order, and possibly also logarithmic, corrections is clear from our results with ferro- and antiferromagnetic $J_{\perp}$. The spin chain in a positive effective staggered field is once again a problem with no continuous symmetry, in which a spin gap opens directly. The key point about this heuristic picture is that the cancellation of the applied and effective staggered fields results in a quasi-1D problem close to $h_{c}$, and hence the anomalous exponents we observe for all values of $J_{\perp}$ are to be expected: $h_{c}$ is always the staggered field required to cancel the $2 \mathrm{D}$ coupling and reduce the model to a $1 \mathrm{D}$ system.

The inexact values of the exponent can be ascribed to departures from universality in the 1D nature of this system, and are are not indicative of a phase genuinely intermediate between 1D and 2D. Because of the effectively 1D nature of the system close to the critical applied staggered field, logarithmic corrections to the gap scaling may remain significant for all values of $\left|J_{\perp}\right|$. We conclude these considerations by commenting on the coupled spinchain system $\mathrm{CDC}, \underline{26}$ where anomalous gap exponents have been measured despite the fact that the interchain coupling is thought to be significant. We suggest on the basis of our results that the origin of this behavior may lie in the effects of a competitive staggered field.

\section{Phase diagram}

Finally, we present in Fig. 11 the phase diagrams of the two competitive-case models for ferro- and antiferromagnetic interchain coupling. The data is taken from Table II. For comparison we show also the chain mean-field results and 2D mean-field results of Ref. [30]. The numerically exact boundary determined by our quantum Monte Carlo simulations lies between those obtained from the two mean-field theories [Fig. [11(b)], showing that neither is particularly accurate for the problem of the $2 \mathrm{D}$ staggered magnetic field, and confirming the general departure from universality of this type of system.

The phase boundary, or critical point as a function of the interchain coupling constant, can be fitted well over the full range of $J_{\perp}$ by a simple power law,

$$
h_{c}=b_{0}\left|J_{\perp}\right|^{\gamma},
$$

with parameters $h_{c}=1.08\left|J_{\perp}\right|^{1.15}$ for the case of ferromagnetic interchain coupling and $h_{c}=1.57\left|J_{\perp}\right|^{1.30}$ for antiferromagnetic coupling.

\section{SUMMARY}

In summary, we have studied the zero-temperature phase diagram and low-energy spin excitations of anisotropic, two-dimensional spin- $\frac{1}{2}$ Heisenberg models on the square lattice under a staggered magnetic field. We have used a continuous-time Monte Carlo method to calculate ordered moments and spin gaps, and hence phase boundaries and scaling relations. At zero field, we compute the properties of the anisotropic Heisenberg 

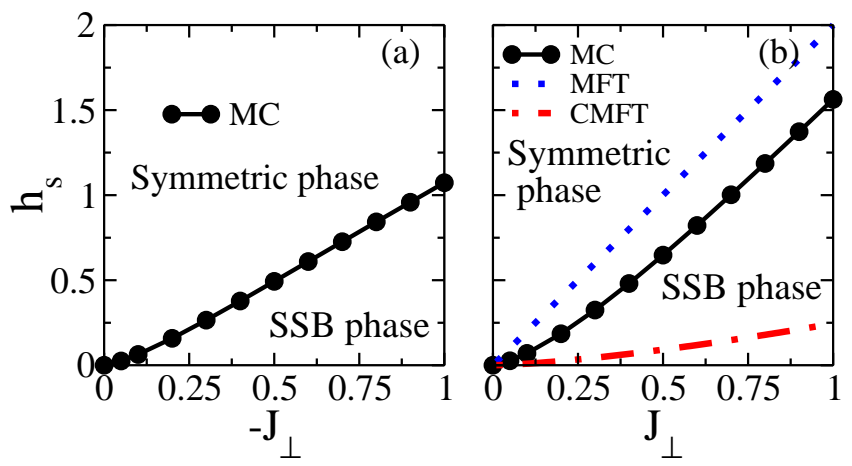

FIG. 11: (Color online) Phase diagrams for competitive-case models in (a) $(\pi, \pi)$ and (b) $(\pi, 0)$ staggered fields, i.e. with (a) ferromagnetic and (b) antiferromagnetic interchain coupling. Monte Carlo results, given in Table [I] are shown by solid circles and power-law fits (see text) by solid lines. In (b), the blue, dotted line marks the phase boundary predicted by a 2D mean-field theory and the red, dashed line that from a chain-based mean-field theory ${ }^{30}$

model and benchmark our calculations for the complexities of high anisotropy and strong logarithmic corrections. When the applied field and magnetic interactions cooperate, a gap opens immediately and scales exactly as the square root of the staggered magnetic field. When the two compete, we find a field-driven quantum phase transition from a gapless phase of staggered magnetic order, and characterized by spontaneous symmetry-breaking in the transverse direction, to a gapped, disordered, fielddominated phase. In the ordered phase, we find a novel enhancement of the staggered moment by a competing field, a purely quantum effect. We determine scaling regimes, discuss the scaling properties of the gap, and show that the physics of the system in the competitive case is essence always one-dimensional at the quantum critical point due to the cancellation of applied and effective staggered fields.

\section{Acknowledgments}

J. Zhao is grateful to N. Prokof'ev for sharing his continuous-time Monte Carlo code. We acknowledge helpful discussions with G. Uhrig. J. Zhao is supported in part by the Japan Society for the Promotion of Science. This work was supported by the National Science Foundation of China under Grant No. 10874244 and by Chinese National Basic Research Project No. 2007CB925001.
1 D. C. Dender, P. R. Hammer, D. H. Reich, C. Broholm, and G. Aeppli, Phys. Rev. Lett 79, 1750 (1997).

2 M. Oshikawa and I. Affleck, Phys. Rev. Lett. 79, 2883 (1997).

3 I. Affleck and M. Oshikawa, Phys. Rev. B 60, 1038 (1999).

4 M. Oshikawa and I. Affleck, Phys. Rev. B 65, 134410 (2002).

${ }^{5}$ F. H. L. Essler and A. M. Tsvelik, Phys. Rev. B 57, 10592 (1998).

${ }^{6}$ F. H. L. Essler, Phys. Rev. B 59, 14376 (1999).

7 F. H. L. Essler, A. Furusaki, and T. Hikihara, Phys. Rev. B 68, 064410 (2003).

8 I. Kuzmenko and F. H. L. Essler, Phys. Rev. B 79, 024402 (2009).

9 A. U. B. Wolter, P. Wzietek, S. Süllow, F. J. Litterst, A. Honecker, W. Brenig, R. Feyerherm, and H. H. Klauss, Phys. Rev. Lett. 94, 057204 (2005).

10 A. U. B. Wolter, H. Rakoto, M. Costes, A. Honecker, W. Brenig, A. Klümper, H. H. Klauss, F. J. Litterst, R. Feyerherm, D. Jerome, and S. Sullow, Phys. Rev. B 68, 220406 (2003).

11 J. Z. Lou, S. J. Qin, C. F. Chen, Z. B. Su, and L. Yu, Phys. Rev. B 65, 064420 (2002).

12 J. Z. Zhao, X. Q. Wang, T. Xiang, Z. B. Su, and L. Yu, Phys. Rev. Lett. 90, 207204 (2003).

13 J. Z. Lou, C. F. Chen, J. Z. Zhao, X. Q. Wang, T. Xiang, Z. B. Su, and L. Yu, Phys. Rev. Lett. 94, 217207 (2005).

14 J. Z. Lou, C. F. Chen, and X. Q. Wang, Phys. Rev. B 73, 092407 (2006).

15 H. Nojiri, Y. Ajiro, T. Asano, and J.-P. Boucher, New J. Phys. 8 218, (2006).

16 Y. Ajiro, T. Asano, Y. Inagaki, J.-P. Boucher, H. Nojiri,
S. Luther, T. Sakon, and M. Motokawa, J. Phys. Soc. Jpn Suppl. A 69, 297 (2000).

17 T. Asano, H. Nojiri, Y. Inagaki, J.-P. Boucher, T. Sakon, Y. Ajiro, and M. Motokawa, Phys. Rev. Lett 84, 5880 (2000).

18 T. Asano, H. Nojiri, W. Higemoto, A. Koda, R. Kadono, and Y. Ajiro, J. Phys. Soc. Jpn 71, 594 (2002).

19 R. Feyerherm, S. Abens, D. Günther, T. Ishida, M. Meissner, M. Meschke, T. Nogami, and M. Steiner, J. Phys.: Condens. Matter 12, 8495 (2000).

20 S. A. Zvyagin, A. K. Kolezhuk, J. Krzystek, and R. Feyerherm, Phys. Rev. Lett. 93, 027201 (2004).

21 S. A. Zvyagin, A. K. Kolezhuk, J. Krzystek, and R. Feyerherm, Phys. Rev. Lett. 95, 017207 (2005).

22 M. Oshikawa, K. Ueda, H. Aoki, A. Ochiai, and M. Kohgi, J. Phys. Soc. Jpn. 68, 3181 (1999).

${ }^{23}$ H. Shiba, K. Ueda, and O. Sakai, J. Phys. Soc. Jpn. 69, 1493 (2000).

24 M. Kohgi, K. Iwasa, J. Mignot, B. Fak, P. Gegenwart, M. Lang, A. Ochiai, H. Aoki, and T. Suzuki, Phys. Rev. Lett. 86, 2439 (2001).

25 I. Umegaki, T. Ono, H. Tanaka, H. Uekusa, and H. Nojiri, unpublished (arXiv:0902.1371).

26 M. Kenzelmann, Y. Chen, C. Broholm, D. H. Reich, and Y. Qiu, Phys. Rev. Lett. 93, 017204 (2004).

27 Y. Chen, M. B. Stone, M. Kenzelmann, C. D. Batista, D. H. Reich, and C. Broholm, Phys. Rev. B 75, 214409 (2007).

28 Y. J. Wang, F. H. L. Essler, M. Fabrizio, and A. A. Nersesyan, Phys. Rev. B 66, 024412 (2002).

29 Y. J. Wang, Phys. Rev. B 68, 214428 (2003).

30 M. Sato and M. Oshikawa, Phys. Rev. B 69, 054406 (2004). 
31 J. Z. Zhao, X. Q. Wang, T. Xiang, Z. B. Su, L. Yu, J. Z. Lou, and C. F. Chen, Phys. Rev. B 73, 012411 (2006).

32 N. V. Prokof'ev, B. V. Svistunov, and I. S. Tupitsyn, JETP Lett. 64, 911 (1996).

${ }^{33}$ N. V. Prokof'ev, B. V. Svistunov, and I. S. Tupitsyn, Phys. Lett. A 238, 253 (1998).

${ }^{34}$ V. A. Kashurnikov, N. V. Prokof'ev, B .V. Svistunov, and M. Troyer, Phys. Rev. B 59, 1162 (1999).

35 T. Oguchi, Phys. Rev. 133, A1098 (1964); T. Ishikawa and T. Oguchi, Prog. Theor. Phys. 54, 1282 (1975).
${ }^{36}$ H.-J. Schulz, Phys. Rev. Lett. 77, 2790 (1996).

37 A. W. Sandvik, Phys. Rev. Lett. 83, 3069 (1999).

38 S. Todo and K. Kato, Phys. Rev. Lett. 87, 047203 (2001).

39 M. P. A. Fisher, P. B. Weichman, G. Grinstein, and D. S. Fisher, Phys. Rev. B 40, 546 (1989).

40 D. A. Huse, Phys. Rev. B 37, 2380 (1988).

41 M. Matsumoto, B. Normand, T. M. Rice, and M. Sigrist, Phys. Rev. B 69, 054423 (2004). 